\title{
SEPARATION BY FINITE SETS \\ IN CONNECTED, CONTINUOUS IMAGES \\ OF ORDERED COMPACTA
}

\author{
L. B. TREYBIG
}

\begin{abstract}
In this paper we answer a question of Mardešic by showing that if the points $x$ and $y$ lie in a continuum $M$ which is the continuous image of a compact ordered space, but $x$ and $y$ lie in no metric subcontinuum of $M$, then $x$ and $y$ are separated in $M$ by a finite set.
\end{abstract}

It is the main purpose of this paper to prove

THEOREM 1. If the Hausdorff continuum $M$ is the image of a compact ordered space $K$ under a continuous map $f$, and $x$ and $y$ are points of $M$ contained in no metric subcontinuum of $M$, then $x$ and $y$ are separated in $M$ by a finite set.

This answers a question of Mardešić [4], except the $K$ in his question was assumed to satisfy the stronger hypothesis of being an arc (nondegenerate ordered continuum). An immediate consequence of Theorem 1 above and Theorem 7 of Pearson [7] (see also [11]) is

THEOREM 2. Under the hypothesis of Theorem 1, if $M$ contains no nondegenerate metric subcontinuum, then $M$ is the continuous image of an arc.

Theorem 2 does give a partial answer (for the case of continua containing no nondegenerate metric subcontinuum) to the following problem of Mardešić and Papić: "Is a connected and locally connected space which is the continuous image of an ordered compactum also the continuous image of an arc?" In the case mentioned above the local connectivity is unnecessary.

In one of the early papers in this area Mardešić [2] showed there is a nondegenerate locally connected Hausdorff continuum which is not arcwise connected. In [1] Cornette and Lehman give a second example, and in [3] Mardešić and Papić show that if a product of two or more nondegenerate continua is the continuous image of an arc, then the product is metrizable. Treybig [8] shows that the connectivity above is not necessary by showing that if a product of two or more infinite compact Hausdorff spaces is the continuous image of a compact ordered space, then the product is metrizable. Pearson shows in [7] that if $H$ is a continuum in which each pair of points is separated by a finite set, then $H$ is the continuous image of an arc. L. E.

Received by the editors May 14, 1978.

AMS (MOS) subject classifications (1970). Primary 54F05, 54F25; Secondary 54B15.

Key words and phrases. Compact ordered space, arc, local connectivity, indecomposable continuum, irreducible continuum, upper semicontinuous decomposition. 
Ward, Jr. proves in [10] that a Hausdorff space is a Peano continuum if and only if it is the strongly irreducible image of some dendrite. Additional results in the area of this paper are contained in [6], [9] and [11].

Proof of Theorem 1. Suppose $x$ and $y$ are not separated in $M$ by a finite subset of $M$. Let $S(x)$ denote the set of all points $p$ of $M$ such that $p$ is not separated from $x$ in $M$ by a finite set. If $z \in M-S(x)$, then there is a finite subset $F$ of $M$ such that $M-F=H_{1} \cup K_{1}$ mutually separated, where $x \in H_{1}$ and $z \in K_{1}$. Since $K_{1}$ is open and no point of $S(x)$ lies in $K_{1}$, then $z$ is not a limit point of $S(x)$. Therefore $S(x)$ is closed. If $S(x)=S_{1} \cup S_{2}$ mutually separated, where $x \in S_{1}$, then there exist disjoint open sets $U, V$ such that $S_{1} \subset U$ and $S_{2} \subset V$. There is a finite collection $K_{1}, K_{2}, \ldots, K_{n}$ of open sets such that

(1) each $K_{i}$ has a finite boundary,

(2) $x \notin \cup_{p=1}^{n} \bar{K}_{p}$, and

(3) $\operatorname{Bd} V \subset \cup_{p=1}^{n} K_{p}$.

Thus $\operatorname{Bd}\left(\cup_{p=1}^{n} K_{p}\right) \cap(M-V)$ is a finite set separating $x$ from a point of $S_{2}$, a contradiction. Therefore, $S(x)$ is a continuum.

Let $C$ be a subcontinuum of $S(x)$ which is irreducible [5] from $x$ to $y$. If $C$ is indecomposable [5], then it is easily seen that no point or pair of points separates $C$, and by Theorem 2 of [9] $C$ is metrizable, a contradiction. Therefore, suppose $C$ is the union of nondegenerate proper subcontinua $C_{1}$, $C_{2}$, where $x \in C_{1}$. Since $C$ is irreducible from $x$ to $y$, then $x \in C_{1}-C_{2}$ and $y \in C_{2}-C_{1}$.

Let $U_{1}, U_{2}, U_{3}, \ldots$ be a sequence of open sets containing $x$ such that $U_{1} \subset M-C_{2}$ and $U_{i+1} \subset \bar{U}_{i+1} \subset U_{i}$ for $i=1,2,3, \ldots$ Let $X=\cap_{1}^{\infty} \bar{U}_{i}$ and let $G$ denote the upper semicontinuous decomposition [5] of $M$ such that $g \in G$ if and only if $g=X$ or $g \in M-X$. If $\phi: M \rightarrow M / G$ denotes the natural map, then $\phi f: K \rightarrow M / G$ is onto and continuous, so by Lemma 4 of [8] there is a compact ordered space $K_{1}$ and continuous onto map $g$ : $K_{1} \rightarrow M / G$ such that $g$ maps no proper closed subset of $K_{1}$ onto $M / G$. It is also easy to verify that (1) $M / G$ satisfies the first axiom of countability at $X$, and (2) no finite subset of $M / G$ separates $X$ from $y$ in $M / G$. Let $S(X)=$ $\left\{g^{\prime}: g^{\prime} \in G\right.$ and no finite subset of $G$ separates $g^{\prime}$ from $X$ in $\left.M / G\right\}$ and note that $C_{2} \subset S(X)$.

For each positive integer $i$ there is a finite cover $Q_{i}$ of $g^{-1}(X)$ such that each element of $Q_{i}$ is of the form $\left\{x: x \in K_{1}\right.$ and $\left.a<x<b\right\}$ or $\left\{x: x \in K_{1}\right.$ and $a<x\}$ or $\left\{x: x \in K_{1}\right.$ and $\left.x<a\right\}$ and is a subset of $g^{-1}\left(U_{i}\right)$. For each such $i$ let $Y_{i}$ denote the set of all points which are an endpoint of some interval of $Q_{i}$, and let $Y=\cup_{1}^{\infty} Y_{i}=\left\{y_{1}, y_{2}, y_{3}, \ldots\right\}$. Also, (see [9]) if $I$ and $J$ are closed subintervals of $K_{1}$ such that $g(I)$ intersects $g(J)$, let $\left(a_{I J}, a_{I J}^{\prime}\right)$, $\left(b_{I J}, b_{I J}^{\prime}\right),\left(c_{I J}, c_{I J}^{\prime}\right)$ and $\left(d_{I J}, d_{I J}^{\prime}\right)$ denote elements of $I \times J$ such that (1) $g\left(x_{I J}\right)=g\left(x_{I J}^{\prime}\right)$ for $x=a, b, c, d$ and (2) if $\left(t, t^{\prime}\right) \in I \times J$ and $g(t)=g\left(t^{\prime}\right)$, then $a_{I J}<t \leqslant b_{I J}$ and $c_{I J}^{\prime} \leqslant t^{\prime} \leqslant d_{I J}^{\prime}$. 
Analogous to the proof of Theorem 2 of [9] let $X_{1}, X_{2}, X_{3}, \ldots$ denote a sequence of finite subsets of $K_{1}=\left[a^{\prime}, b^{\prime}\right]$ such that (1) $a^{\prime}, b^{\prime} \in X_{1}$ and $g\left(X_{1}\right)$ contains at least three points of $C_{2}$, (2) for each positive integer $n$, $\left\{y_{1}, y_{2}, \ldots, y_{n}\right\} \subset X_{n} \subset X_{n+1}$, and if each of $p, q, r, s$ is an element of $X_{n}$ and $I=[p, q]$ and $J=[r, s]$ are subintervals of $K_{1}$ such that $g(I)$ intersects $g(J)$, then $X_{n+1}$ contains $x_{I J}$ and $x_{I J}^{\prime}$ for $x=a, b, c, d$.

Let $Q$ denote the set of all open subsets of $K_{1}$ which are maximal with respect to the property of being convex subsets of $K_{1}$ which do not intersect $\mathrm{Cl}\left(\cup_{1}^{\infty} X_{i}\right)$. Suppose $(r, s) \in Q$, and $w \in g^{-1}\left(C_{2}\right)$ such that $r<w<s$ and $g(w) \notin g\left(\operatorname{Cl}\left(\cup_{1}^{\infty} X_{i}\right)\right)$. Let $H=\{q \in Q$ : there is a finite sequence $q_{0}, q_{1}, \ldots, q_{n}$ such that $q_{0}=(r, s), q_{n}=q$, and $g\left(q_{i}\right)$ intersects $g\left(q_{i+1}\right)$ for $i=0, \ldots, n-1\}$. By [9] the following hold: If $(u, v) \in H$ then $\{g(u), g(v)\}$ $\subset\{g(r), g(s)\}$; and if $(t, u) \in H$ and $z_{1}, z_{2} \in K_{1}$ such that $z_{1} \in \mathrm{Cl}\left(\cup_{1}^{\infty} X_{i}\right)$, $z_{2} \in(t, u)$, and $g\left(z_{1}\right)=g\left(z_{2}\right)$, then $g\left(z_{2}\right) \in\{g(r), g(s)\}$.

As in the proof of Theorem 2 of [9] it follows that $M / G$ is the union of two closed sets $L_{1}=g(\cup H) \cup\{g(r), g(s)\}, L_{2}=g\left(K_{1}-\cup H\right) \cup\{g(r), g(s)\}$, where $L_{1} \cap L_{2}=\{g(r), g(s)\}$. Since $Y \subset \cup_{1}^{\infty} X_{i}$, it follows that $X \notin$ $\{g(r), g(s)\}$, but $g(w) \in L_{1}-\{g(r), g(s)\}$ and $C_{2}$ also intersects $L_{2}-$ $\{g(r), g(s)\}$. Therefore $\{g(r), g(s)\}$ separates $X$ from a point of $S(X)$ in $M / G$, a contradiction. It follows that $C_{2} \subset g\left(\mathrm{Cl}\left(\cup_{1}^{\infty} X_{i}\right)\right)$, and Lemma 2 of [8] implies that $g^{-1}\left(C_{2}\right) \cap \mathrm{Cl}\left(\cup_{1}^{\infty} X_{i}\right)$ is separable. Theorem 1 of [9] implies that $C_{2}$ is metrizable. Analogously, $C_{1}$ is metrizable, so $C=C_{1} \cup C_{2}$ is also, a contradiction. Therefore, $x$ and $y$ are separated in $M$ by a finite subset of M.

\section{REFERENCES}

1. J. L. Cornette and B. Lehman, Another locally connected Hausdorff continuum not connected by ordered continua, Proc. Amer. Math. Soc. 35 (1972), 281-284.

2. S. Mardesic, On the Hahn-Mazurkiewicz theorem in nonmetric spaces, Proc. Amer. Math. Soc. 11 (1960), 927-937.

3. S. Mardešić and P. Papić, Continuous images of ordered continua, Glasnik Mat.-Fiz. Astronom. 15 (1960), 171-178.

4. S. Mardešic, Problem M1, Proc. Internat. Sympos. on Topology and Its Appl. (HercegNovi, 1968), Savez Drustava Mat. Fiz. i Astronom., Belgrade, 1969, p. 349.

5. R. L. Moore, Foundations of point set theory, Amer. Math. Soc. Colloq. Publ., vol. 13, Amer. Math. Soc., Providence, R.I., 1932.

6. B. J. Pearson, Mapping an arc onto a dendritic continuum, Colloq. Math. 30 (1974), 237-243.

7. _ Mapping arcs and dendritic spaces onto netlike continua, Colloq. Math. 34 (1975), 39-48.

8. L. B. Treybig, Concerning continuous images of compact ordered spaces, Proc. Amer. Math. Soc. 15 (1964), 866-871.

9. Concerning continua which are continuous images of compact ordered spaces, Duke Math. J. 32 (1965), 417-422.

10. L. E. Ward, Jr., An irreducible Hahn-Mazurkiewicz theorem (preprint).

11. The Hahn-Mazurkiewicz theorem for rim finite continua, General Topology and Appl. 6 (1976), 183-190.

Department of Mathematics, Texas A\&M University, College Station, Texas 77843 